\title{
Tracking oseltamivir-resistance in New Zealand influenza viruses during a medicine reclassification in 2007, a resistant-virus importation in 2008 and the 2009 pandemic
}

\author{
Richard J Hall, a Matthew Peacey, ${ }^{a}$ Jacqui C. Ralston, ${ }^{a}$ Danielle J de Joux, ${ }^{a}$ Judy Bocacao, ${ }^{a}$ Mackenzie Nicol, ${ }^{a}$
} Molly Ziki, ${ }^{a}$ Wendy Gunn, ${ }^{a}$ Jing Wang ${ }^{a}$ and Q Sue Huang ${ }^{\circ}$

Correspondence to Richard J Hall (e-mail: richard.hall@esr.cri.nz).

Introduction: Oseltamivir (Tamifluß) is an important pharmaceutical intervention against the influenza virus. The importance of surveillance for resistance to oseltamivir has been highlighted by two global events: the emergence of an oseltamivir-resistant seasonal influenza $A(H 1 N 1)$ virus in 2008 , and emergence of the influenza $A(H 1 N 1) p d m 09$ virus in 2009. Oseltamivir is a prescription medicine in New Zealand, but more timely access has been provided since 2007 by allowing pharmacies to directly dispense oseltamivir to patients with influenza-like illness.

Objective: To determine the frequency of oseltamivir-resistance in the context of a medicine reclassification in 2007, the importation of an oseltamivir-resistant seasonal influenza virus in 2008, and the emergence of a pandemic in 2009.

Methods: A total of 1795 influenza viruses were tested for oseltamivir-resistance using a fluorometric neuraminidase inhibition assay. Viruses were collected as part of a sentinel influenza surveillance programme between the years 2006 and 2010.

Results: All influenza B, influenza $A(H 3 N 2)$ and influenza $A(H 1 N 1) p d m 09$ viruses tested between 2006 and 2010 were shown to be sensitive to oseltamivir. Seasonal influenza $A(H 1 N 1)$ viruses from 2008 and 2009 were resistant to oseltamivir. Sequencing of the neuraminidase gene showed that the resistant viruses contained an H275Y mutation, and S247N was also identified in the neuraminidase gene of one seasonal influenza $A(H 1 N 1)$ virus that exhibited enhanced resistance.

Discussion: No evidence was found to suggest that increased access to oseltamivir has promoted resistance. A probable importation event was documented for the global 2008 oseltamivir-resistant seasonal $A(H 1 N 1)$ virus nine months after it was first reported in Europe in January 2008.

$\mathrm{O}$ ver the last decade there has been an extensive amount of research into the development and occurrence of antiviral drug resistance in human influenza viruses. ${ }^{1}$ An effective class of anti-influenza drugs known as neuraminidase inhibitors have been developed which include the drug oseltamivir phosphate (Tamiflu®). Neuraminidase inhibitors block the release of progeny virions from a host cell by selectively binding to the active site of the neuraminidase enzyme. This inhibits cleavage of the sialyl-acid bond to the host receptor, thus the virus is unable to be released from infected host cells and spread to new cells. ${ }^{2}$ Clinical trials of oseltamivir have shown reduced symptom severity and a reduction in the duration of the illness. ${ }^{3-5}$ Oseltamivir is reported to be widely used, with 65 million treatment courses prescribed worldwide. ${ }^{6}$ Oseltamivir-resistance in influenza should be closely monitored to determine if the continued efficacy of oseltamivir warrants its use for influenza. ${ }^{7}$ Such work not only determines the present efficacy of the drug but also reveals important information on the genesis of anti-viral drug resistance in influenza viruses.

In New Zealand, oseltamivir is a prescription medicine that is most effective if administered within the first 48 hours of infection. In 2007, to increase availability of oseltamivir and reduce delays in obtaining a prescription of oseltamivir from a medical doctor,

\footnotetext{
WHO National Influenza Centre, Institute of Environmental Science \& Research, National Centre for Biosecurity \& Infectious Disease, New Zealand. Submitted: 9 August 2012; Published: 10 December 2012 doi: 10.5365/wpsar.2012.3.3.002
} 
pharmacists were allowed to directly provide oseltamivir during the winter influenza season (April to November inclusive). The pharmacist had to be satisfied that the oseltamivir was for a resident of New Zealand, aged 12 years or more and presenting with the symptoms of influenza. ${ }^{8}$ This allowance was made with an expectation that influenza viruses from the community would be monitored for the potential development of oseltamivirresistance. ${ }^{9}$

Preceding the 2007/2008 northern hemisphere season, instances of oseltamivir-resistance occurred at low levels in seasonal human influenza viruses. ${ }^{10}$ Increased occurrence of resistance in influenza $\mathrm{A}(\mathrm{H} 1 \mathrm{~N} 1) p d m 09$ viruses has been detected in community samples in the United Kingdom, ${ }^{11}$ and sustained community transmission has been reported in Australia. ${ }^{12}$ Resistance has been shown to be caused by a number of mutations, particularly the His275Tyr (N1 numbering; herein referred to as $\mathrm{H} 275 \mathrm{Y}$ ) of the neuraminidase (NA) gene in influenza $A(H 1 N 1)$ viruses. ${ }^{13}$ In the winter of 2007/2008, a relatively high incidence of resistant seasonal $A(\mathrm{H} 1 \mathrm{~N} 1)$ influenza viruses was detected in Europe (average 20\%). ${ }^{14,15}$ These resistant viruses, which were shown to carry an $\mathrm{H} 275 \mathrm{Y}$ mutation, were subsequently reported in many other regions of the world. ${ }^{16-18}$

In this study we monitored the frequency of oseltamivir-resistance in influenza viruses circulating in New Zealand between 2006 and 2010. This surveillance was performed during a series of events that had the potential to alter the resistance profiles of circulating influenza viruses, including a change in the availability of oseltamivir at pharmacies in 2007 , the importation of oseltamivir-resistant seasonal influenza viruses in 2008 , and the emergence of influenza A(H1N1)pdm09. We discuss these findings in relation to the genesis of antiviral drug resistance in New Zealand, the importance of surveillance and in relation to findings overseas.

\section{METHODS}

\section{Clinical material}

Clinical samples were collected in New Zealand as part of the national influenza sentinel surveillance programme, which has been previously described. ${ }^{19,20}$ Briefly, samples were collected weekly from general medical practice patients presenting with influenza-like illness, defined as an acute respiratory tract infection characterized by the abrupt onset of at least two of the following: fever, chills, headache and myalgia. Nasopharyngeal swabs or throat swabs were taken from patients and transported to the laboratory in viral transport media. Samples from 2006 to 2008 were obtained during the winter influenza season from May to September. Samples from 2009 and 2010 were obtained over the entire year as influenza surveillance was extended due to the pandemic. ${ }^{21}$ Additional clinical samples were obtained from hospital diagnostic laboratories in New Zealand throughout the course of each year as part of a reference testing service. These hospitals were located in Auckland, Waikato, Christchurch and Dunedin.

Diagnosis of influenza virus was made either by real-time reverse transcriptase polymerase chain reaction (RT-PCR) (method developed by Centers of Disease Control and Prevention, Atlanta, Georgia; World Health Organization [WHO] recommended), or viral culture followed by a haemagglutination/haemagglutinationinhibition assay using WHO reference antisera.

\section{Viral culture}

Influenza viruses from 2006 to 2008 were grown in cultured Madin-Darby Canine Kidney (MDCK) cells in serum-free M199 media in the presence of TPCK-trypsin. Influenza viruses from 2009 were also grown in the cultured MDCK-SIAT1 cell line in DMEM:F12 media in the presence of TPCK-trypsin. ${ }^{22}$

\section{Fluorometric neuraminidase-inhibition assay}

Sensitivity of influenza viruses to oseltamivir was determined by fluorometric neuraminidase-inhibition assay, as described previously. ${ }^{23,24}$ Briefly, neuraminidase was solubilised from influenza virus culture in a buffer containing $1 \% \mathrm{NP}-40$ at a ratio of $1: 10$ for seasonal influenza viruses or 1:20 for influenza $A(\mathrm{H} 1 \mathrm{~N} 1)$ pdm09 viruses. The activity of neuraminidase was measured over a range of concentrations of oseltamivir carboxylate (obtained from Roche, Switzerland) with the fluorogenic substrate MUNANA at $37^{\circ} \mathrm{C}$ for one hour and then fluorescence was measured (emission $360 \mathrm{~nm}$; excitation $448 \mathrm{~nm}$ ). The 50\% inhibitory concentration $\left(\mathrm{IC}_{50}\right)$ was determined using a log- 
dose-response curve-fit in GraphPad PRISM (v5.04). The $\mathrm{IC}_{50}$ value represented the concentration at which oseltamivir inhibits neuraminidase activity by $50 \%$. For any outliers that were initially identified as having $\mathrm{IC}_{50}$ values greater than two standard deviations above the mean, a titration of the virus was performed to ensure viral dilution was at the mid-point of the linear range of neuraminidase activity. Adjusted $I_{50}$ values for these initial outliers were recalculated as required and included in the final analysis. A greater than 10 -fold increase from the mean $I_{50}$ value within a subtype was taken to be indicative of oseltamivirresistance. ${ }^{25}$

\section{Sequencing of the neuraminidase gene}

The viral RNA was extracted directly from the clinical specimen using the Zymo viral RNA extraction kit (Zymo Research, Irvine California, United States of America; cat\# R1034). The entire NA gene was amplified using universal NA influenza primers ${ }^{26}$ and the same primers were used for direct sequencing by the Sanger method (Big Dye Terminator v.3.1 cycle sequencing kit, Applied Biosystems, Nieuwerkerk, NL) on a capillary sequencer (Model 3100 Avant, Applied Biosystems, Foster City, California, United States of America).

\section{Sales data for oseltamivir}

Sales data for oseltamivir in 2004 and 2007 was provided courtesy of Roche Pharmaceuticals. Data include both prescription and pharmacy-exemption sales.

\section{Ethical statement}

Samples were obtained as part of public health surveillance. Clinical conduct was consistent with the New Zealand Code of Health and Disability Services Consumers' Rights.

\section{RESULTS}

A total of 1795 influenza samples collected in New Zealand between 2006 and 2010 were tested for sensitivity to oseltamivir by fluorometric neuraminidase inhibition assay (Figure 1, Table 1).

All 521 influenza A viruses and all 133 influenza B viruses from 2006 and 2007 were shown to be sensitive to oseltamivir. In 2008, 306 influenza B and 120 influenza $A(H 3 N 2)$ viruses were found to be sensitive to oseltamivir. However, all four seasonal influenza $A(H 1 N 1)$ viruses isolated in this year were

Table 1. Comparison of $I_{C_{50}}$ values $^{*}$ for influenza viruses from New Zealand isolated from 2006 to 2010

\begin{tabular}{|c|c|c|c|c|c|c|}
\hline \multirow{2}{*}{\multicolumn{2}{|c|}{ Influenza type/subtype }} & \multicolumn{5}{|c|}{ Year } \\
\hline & & 2006 & 2007 & 2008 & 2009 & 2010 \\
\hline \multirow[t]{6}{*}{ B } & Number of isolates tested & 1 & 132 & 306 & - & 1 \\
\hline & Mean $\mathrm{IC}_{50}(\mathrm{nM})$ & - & 37.5 & 26.5 & - & - \\
\hline & Standard deviation (nM) & - & 22.5 & 16.9 & - & - \\
\hline & Minimum $\mathrm{IC}_{50}(\mathrm{nM})$ & - & 0.9 & 0.2 & - & - \\
\hline & Maximum $\mathrm{IC}_{50}(\mathrm{nM})$ & - & 97.4 & 87.8 & - & - \\
\hline & Oseltamivir-resistance detected & - & No & No & - & No \\
\hline \multirow[t]{6}{*}{$\mathrm{A}(\mathrm{H} 3 \mathrm{~N} 2)$} & Number of isolates tested & 189 & 45 & 120 & - & 1 \\
\hline & Mean IC ${ }_{50}(\mathrm{nM})$ & 0.7 & 0.4 & 0.3 & - & - \\
\hline & Standard deviation (nM) & 0.3 & 0.3 & 0.2 & - & - \\
\hline & Minimum $\mathrm{IC}_{50}(\mathrm{nM})$ & 0.1 & 0.1 & 0.1 & - & - \\
\hline & Maximum $\mathrm{IC}_{50}(\mathrm{nM})$ & 1.4 & 1.1 & 1.1 & - & - \\
\hline & Oseltamivir-resistance detected & No & No & No & - & No \\
\hline \multirow[t]{6}{*}{ A(H1N1) } & Number of isolates tested & 18 & 136 & 4 & 25 & - \\
\hline & Mean $\mathrm{IC}_{50}(\mathrm{nM})$ & 1.3 & 0.8 & 768 & 1385 & - \\
\hline & Standard deviation (nM) & 0.9 & 0.6 & 287 & 1996 & - \\
\hline & Minimum $\mathrm{IC}_{50}(\mathrm{nM})$ & 0.2 & 0.1 & 573 & 305 & - \\
\hline & Maximum $\mathrm{IC}_{50}(\mathrm{nM})$ & 3.0 & 2.7 & 1184 & 7912 & - \\
\hline & Oseltamivir-resistance detected & No & No & Yes & Yes & - \\
\hline \multirow[t]{6}{*}{ A(H1N1)pdm09 } & Number of isolates tested & - & - & - & 483 & 334 \\
\hline & Mean $I_{50}(n M)$ & - & - & - & 0.4 & 0.7 \\
\hline & Standard deviation (nM) & - & - & - & 0.2 & 0.4 \\
\hline & Minimum $\mathrm{IC}_{50}(\mathrm{nM})$ & - & - & - & 0.1 & 0.1 \\
\hline & Maximum $\mathrm{IC}_{50}(\mathrm{nM})$ & - & - & - & 1.4 & 2.1 \\
\hline & Oseltamivir-resistance detected & - & - & - & No & No \\
\hline
\end{tabular}

* $\mathrm{IC}_{50}$ - inhibitory concentration of the drug at which a $50 \%$ reduction in enzymatic activity is observed. 
Figure 1. Box plots of Log10 IC ${ }_{50}$ values* for each influenza virus type, New Zealand, 2006 to $2010^{\dagger}$
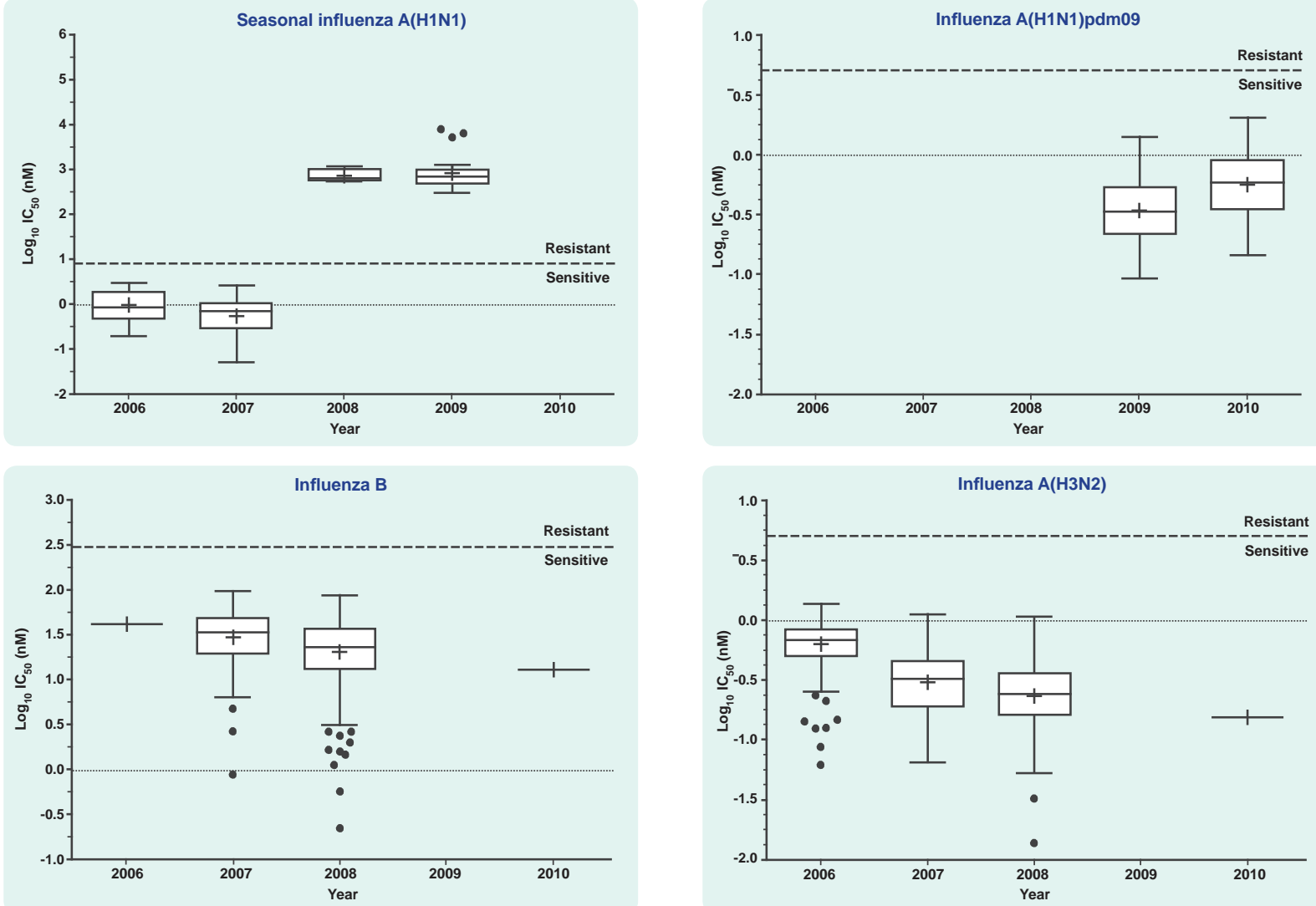

$\mathrm{IC}_{50}$ - inhibitory concentration of the drug at which a $50 \%$ reduction in enzymatic activity is observed.

Boxes indicate the first, second and third quartiles and the whiskers are calculated as 1.5 times the interquartile distance. Outliers beyond this distance are plotted individually as $(\bullet)$, and the mean for each data set is indicated by the symbol $(+)$. The thick dashed line represents the threshold at which viruses are determined to be resistant to oseltamivir. This threshold is 10 -fold higher than the mean $I_{50}$ value for all years, calculated for each subtype. The calculation of the mean $\mathrm{IC}_{50}$ value threshold does not include 2008 and 2009 seasonal influenza $\mathrm{A}(\mathrm{H} 1 \mathrm{~N} 1)$ viruses, as all viruses in these years were resistant to oseltamivir. The thin dashed line shows the $\log 10 \mathrm{IC}_{50}$ zero axis.

Figure 2. Sales data for oseltamivir, New Zealand, 2004 and 2007

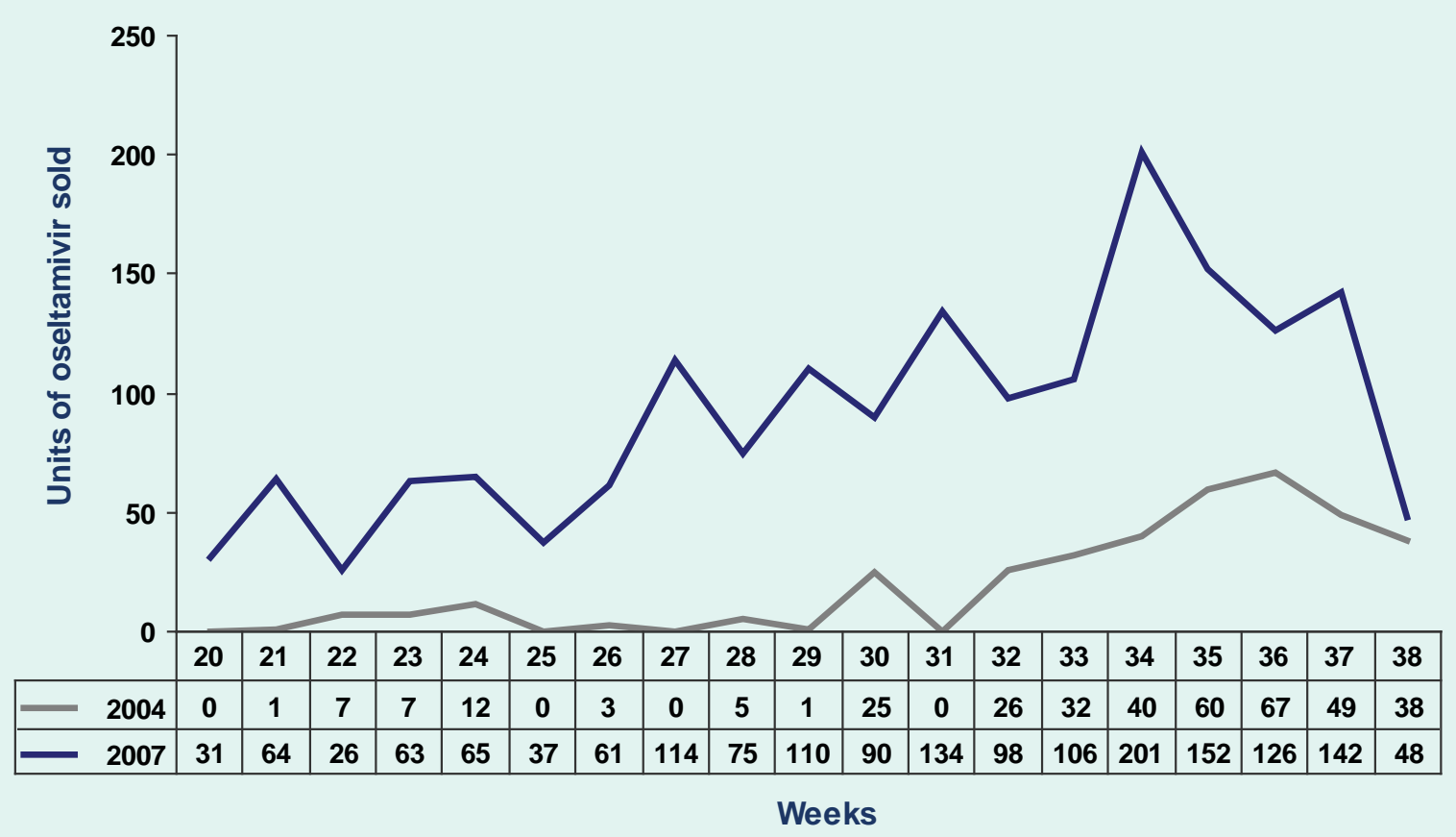


resistant to oseltamivir with $\mathrm{IC}_{50}$ values between 573 nM and 1184 nM (Figure 1, Table 1). Full-length sequencing of the NA gene for two of these viruses (sequence coverage of nucleotides 21-1413 and 22-940) revealed the presence of the $\mathrm{H} 275 \mathrm{Y}$ mutation, with the sequenced region having almost complete identity ( $99 \%$ and $100 \%$ respectively) to the 2008 resistant-type viruses that had been reported from Europe earlier in the year [GenBank Accession: EU566977; A/Pennsylvania/02/2008(H1N1)]. Only a single nucleotide difference was observed (substitution E268D; G/T nucleotide 804; N1 subtype numbering).

As we have previously reported, ${ }^{27}$ all 2009 seasonal $A(H 1 N 1)$ viruses tested for sensitivity to oseltamivir were resistant ( $n=25$; Figure 1 ; Table 1 ), with $I_{50}$ values between 305 nM and 7912 nM. ${ }^{27}$ All were also shown to contain the H275Y mutation by RFLP analysis or by sequencing. Further sequencing of the NA gene of the $7912 \mathrm{nM}$ virus (A/Wellington/31/2009), one of the three seasonal influenza $A(H 1 N 1)$ viruses with extremely high $\mathrm{IC}_{50}$ values of $5334 \mathrm{nM}, 6370 \mathrm{nM}$ and $7912 \mathrm{nM}$ in this study (Figure 1 ), identified an additional significant mutation S247N (N1 numbering), as well as $\mathrm{H} 275 \mathrm{Y}$ (GenBank accession KC117387).

All influenza $A(\mathrm{H} 1 \mathrm{~N} 1)$ pdm09 viruses tested in this study from 2009 and 2010 were shown to be sensitive to oseltamivir (Figure 1, Table 1 ) and sequencing of the NA gene for 11 of these viruses found that none carried the H275Y mutation.

Oseltamivir sales data in New Zealand for 2004 and 2007 showed a 4.5-fold increase in usage with 373 doses sold in 2004 compared to 1678 doses sold in 2007 (Figure 2). The greatest difference between 2004 and 2007 was in week 34 with 161 more units of oseltamivir sold in 2007 compared to 2004. As the population of New Zealand is 4.5 million, this increase in usage represents only an extremely small proportion of the total population.

\section{DISCUSSION}

This study shows that antiviral drug resistance to oseltamivir between 2006 and 2010 occurred at a very low level for most human influenza viruses in New Zealand. The exceptions to this observation were the seasonal $A(H 1 N 1)$ viruses from January 2008 onward, which showed high levels of resistance.
This virus appears to have arrived in New Zealand (a southern hemisphere country) during the winter influenza season, nine months after its emergence was first reported in Europe. ${ }^{14,15}$ Other southern hemisphere countries such as Australia, South Africa and South America also reported the emergence of oseltamivirresistant seasonal influenza viruses late in $2008 .^{17,28}$ The resistant-type seasonal $A(H 1 N 1)$ became the predominant influenza virus in the first half of the New Zealand 2009 influenza season, showing that this virus is capable of both sustained transmission in the community and maintaining resistance to oseltamivir. ${ }^{29}$ Interestingly, we also note the occurrence of three of these seasonal $A(H 1 N 1)$ viruses with extremely high resistance to oseltamivir, brought about by the dual mutations $\mathrm{S} 247 \mathrm{~N}+\mathrm{H} 275 \mathrm{Y}$. The S247N mutation reportedly reduces sensitivity to oseltamivir in seasonal $A(H 1 N 1)$ viruses ${ }^{30}$ and influenza $A(H 5 N 1)$ viruses $^{31}$ and is known to cause extreme resistance to oseltamivir in influenza $\mathrm{A}(\mathrm{H} 1 \mathrm{~N} 1)$ pdm09 viruses in combination with H275Y. ${ }^{32}$ Our results indicate that the presence of the dual $\mathrm{S} 247 \mathrm{~N}+\mathrm{H} 275 \mathrm{Y}$ mutation is likely to have a similar effect in seasonal $A(H 1 N 1)$ viruses.

Before 2008, we observed no oseltamivir-resistance for any influenza type/subtype in New Zealand. This is despite the regulatory change for oseltamivir in New Zealand in 2007, where it could be prescribed by pharmacists to patients presenting with influenza-like illness during the winter influenza season. ${ }^{8}$ A similar system was established in the United Kingdom where accredited pharmacists were able to supply oseltamivir to at-risk individuals during influenza outbreaks. ${ }^{33}$ Increased public access to the drug raises the potential for drug resistance due to selective pressure on the virus in individual patients undergoing treatment. ${ }^{34}$ However, since no substantial increase in usage in New Zealand was observed between 2004 and 2007, we cannot speculate what impact the medicine reclassification had on oseltamivir resistance. A comparative study in Japan, where oseltamivir is more widely used, reported no significant effect on the occurrence of resistance. ${ }^{35}$

Oseltamivir is important for controlling the transmission and dissemination of pandemic viruses before a vaccine becomes widely available. No vaccine was available in New Zealand until one year after the first cases of influenza $\mathrm{A}(\mathrm{H} 1 \mathrm{~N} 1)$ pdm09 arose. This study shows that $100 \%$ of 817 influenza $A(H 1 N 1)$ 
pdm09 viruses from 2009 and 2010 were sensitive to oseltamivir. During the early phases of the pandemic, New Zealand health authorities deployed a percentage of the pandemic stockpile of oseltamivir $(<50000$ doses; Ministry of Health, New Zealand Government), which likely assisted in the initial containment of the pandemic. It took approximately six to seven weeks from the first reported New Zealand cases on 26 April 2010 to the declaration of management phase in June 2010 when the virus had established community transmission. Previous epidemiological modelling studies have suggested that increased usage of oseltamivir during a pandemic may trigger the development of resistant viruses with no reduction in fitness to the virus. ${ }^{36,37}$ The levels of oseltamivir used in New Zealand are unlikely to have approached thresholds developed in these modelling studies, but our data show that oseltamivir-resistance in influenza $A(H 1 N 1) p d m 09$ viruses remained low despite the issuance of pandemic stockpiles of oseltamivir.

Continued surveillance for anti-viral drug resistance in influenza viruses is still required to ensure that stockpiled neuraminidase-inhibitors are effective and that clinicians can be kept informed of the efficacy of neuraminidase inhibitors when treating patients for influenza.

\section{Conflicts of interest}

None declared.

\section{Funding}

This work was funded in part by the New Zealand Ministry of Health and New Zealand Ministry of Science and Innovation.

\section{Acknowledgements}

New Zealand national influenza surveillance is funded by the Ministry of Health who kindly permitted the use of relevant data for publication. We also thank Darren Hunt for his review of the manuscript. Our special thanks to the GPs and nurses, the public health unit coordinators and the participating virology laboratories in Auckland, Christchurch, Waikato and National Influenza Centre at ESR. We also wish to acknowledge the kind support, advice and guidance from Aeron Hurt and
Ian Barr at the WHO Collaborating Centre for Reference and Research on Influenza, Melbourne, Australia. We acknowledge Roche Pharamceuticals for providing sales data on oseltamivir which is presented in this study. We also thank the CDC for sharing the influenza virus RT-PCR protocol through a material transfer agreement. The MDCK-SIAT1 cells were a gift from M Matrosovich, Philipps University, Marburg.

\section{References:}

1. Hurt AC, Ho HT, Barr I. Resistance to anti-influenza drugs: adamantanes and neuraminidase inhibitors. Expert Review of Anti-Infective Therapy, 2006, 4:795-805. doi: 10.1586/14787210.4.5.795 pmid:17140356

2. Matrosovich $\mathrm{MN}$ et al. Neuraminidase is important for the initiation of influenza virus infection in human airway epithelium. Journal of Virology, 2004, 78:12665-12667. doi:10.1128/ JVI.78.22.12665-12667.2004 pmid:15507653

3. Aoki FY et al.; IMPACT Study Group. Early administration of oral oseltamivir increases the benefits of influenza treatment. Journal of Antimicrobial Chemotherapy, 2003, 51:123-129. doi:10.1093/ jac/dkg007 pmid:12493796

4. Nicholson KG et al.; Neuraminidase Inhibitor Flu Treatment Investigator Group. Efficacy and safety of oseltamivir in treatment of acute influenza: a randomised controlled trial. The Lancet, 2000, 355: 1845-1850. doi:10.1016/S01406736(00)02288-1 pmid:10866439

5. Treanor JJ et al.; US Oral Neuraminidase Study Group. Efficacy and safety of the oral neuraminidase inhibitor oseltamivir in treating acute influenza: a randomized controlled trial. JAMA: The Journal of the American Medical Association, 2000, 283:1016-1024. doi:10.1001/jama.283.8.1016 pmid:10697061

6. Dutkowski R. Oseltamivir in seasonal influenza: cumulative experience in low- and high-risk patients. Journal of Antimicrobial Chemotherapy, 2010, 65 Suppl 2:ii11-24. doi:10.1093/jac/ dkq012 pmid:20215131

7. Hurt AC et al.; WHO Consultation on Pandemic Influenza A(H1N1) 2009 Virus Resistance to Antivirals. Antiviral resistance during the 2009 influenza A H1N1 pandemic: public health, laboratory, and clinical perspectives. The Lancet Infectious Diseases, 2012, 12:240-248. doi:10.1016/S1473-3099(11)70318-8 pmid:22186145

8. Minutes of the 34th meeting of the Medicines Classification Committee. Auckland, New Zealand Medicines Classification Committee, $2006 \quad$ (http://www.medsafe.govt.nz/profs/class/ mccMin9June06.htm, accessed 28 November 2012).

9. Oseltamivir Report 2006. Wellington, New Zealand Medicines and Medical Safety Devices Authority, 2007.

10. Monto AS et al. Detection of influenza viruses resistant to neuraminidase inhibitors in global surveillance during the first 3 years of their use. Antimicrobial Agents and Chemotherapy, 2006, 50:2395-2402. doi:10.1128/AAC.01339-05 pmid: 16801417

11. Lackenby $A$ et al. Continued emergence and changing epidemiology of oseltamivir-resistant influenza A(H1N1)2009 virus, United Kingdom, winter 2010/11. Euro Surveillance: European Communicable Disease Bulletin, 2011, 16(6):19789. pmid:21315056

12. Hurt $A C$ et al. Community transmission of oseltamivir-resistant A(H1N1)pdm09 influenza. The New England Journal of 
Medicine, 2011, 365:2541-2542. doi:10.1056/ NEJMc1111078 pmid:22204735

13. Yen $\mathrm{HL}$ et al. Importance of neuraminidase active-site residues to the neuraminidase inhibitor resistance of influenza viruses. Journal of Virology, 2006, 80:8787-8795. doi:10.1128/ JVI.00477-06 pmid:16912325

14. Lackenby $A$ et al. Emergence of resistance to oseltamivir among influenza $A(\mathrm{H} 1 \mathrm{~N} 1)$ viruses in Europe. Euro Surveillance: European Communicable Disease Bulletin, 2008, 13:8026. pmid: 18445375

15. Meijer A et al.; European Influenza Surveillance Scheme. Oseltamivir-resistant influenza virus $A(H 1 N 1)$, Europe, 2007-08 season. Emerging Infectious Diseases, 2009, 15: 552-560. doi:10.3201/eid1504.081280 pmid:19331731

16. Dharan NJ et al.; Oseltamivir-Resistance Working Group. Infections with oseltamivir-resistant influenza $\mathrm{A}(\mathrm{H} 1 \mathrm{~N} 1)$ virus in the United States. JAMA: The Journal of the American Medical Association, 2009, 301:1034-1041. doi:10.1001/ jama.2009.294 pmid:19255110

17. Hurt $A C$ et al. Emergence and spread of oseltamivir-resistant $A(H 1 N 1)$ influenza viruses in Oceania, South East Asia and South Africa. Antiviral Research, 2009, 83:90-93. doi:10.1016/j.antiviral.2009.03.003 pmid:19501261

18. Influenza A virus resistance to oseltamivir - 13 June 2008 Geneva, World Health Organization, 2008 ( http://www.who.int/ influenza/patient_care/antivirals/oseltamivir_summary/en/index. html, accessed $2 \overline{8}$ November 2012).

19. Huang QS et al. Influenza surveillance and immunisation in New Zealand, 1997-2006. Influenza and Other Respiratory Viruses, 2008, 2:139-145. doi:10.1111/j.1750-2659.2008. 00050.x pmid: 19453466

20. Huang QS, Lopez L, Adlam B. Influenza surveillance in New Zealand in 2005. New Zealand Medical Journal, 2007 120:U2581. pmid:17589549

21. Huang $Q$ et al. Surveillance for the 2009 pandemic influenza $A(H 1 N 1)$ virus and seasonal influenza viruses - New Zealand, 2009. MMWR, Morbidity and Mortality Weekly Report, 2009, 58:918-921.

22. Matrosovich $M$ et al. Overexpression of the alpha-2, 6-sialyltransferase in MDCK cells increases influenza virus sensitivity to neuraminidase inhibitors. Journal of Virology, 2003 , 77:8418-8425. doi:10.1128/JVI.77.15.8418-8425.2003 pmid:12857911

23. Ives $\mathrm{JA}$ et al. The $\mathrm{H} 274 \mathrm{Y}$ mutation in the influenza A/H1N1 neuraminidase active site following oseltamivir phosphate treatment leave virus severely compromised both in vitro and in vivo. Antiviral Research, 2002, 55:307-317. doi:10.1016/S0166-3542(02)00053-0 pmid:12103431

24. Wetherall NT et al. Evaluation of neuraminidase enzyme assays using different substrates to measure susceptibility of influenza virus clinical isolates to neuraminidase inhibitors: report of the neuraminidase inhibitor susceptibility network. Journal of Clinical Microbiology, 2003, 41:742-750. doi:10.1128/ JCM.41.2.742-750.2003 pmid:12574276

25. Zambon M, Hayden FG, Global Neuraminidase Inhibitor Susceptibility N. Position statement: global neuraminidase inhibitor susceptibility network. Antiviral Research, 2001, 49:147-156. doi:10.1016/S0166-3542(01)00124-3 pmid:11428241

26. Hoffmann $E$ et al. Universal primer set for the full-length amplification of all influenza A viruses. Archives of Virology, 2001 , 146:2275-2289. doi:10.1007/s007050170002 pmid: 11811679

27. Hall RJ et al. Pandemic influenza $A(H 1 N 1)$ viruses currently circulating in New Zealand are sensitive to oseltamivir. Euro Surveillance: European Communicable Disease Bulletin, 2009, 14:19282. pmid:19643060

28. García J et al. Antiviral resistance in influenza viruses circulating in Central and South America based on the detection of established genetic markers. Influenza and Other Respiratory Viruses, 2009, 3:69-74. doi:10.1111/j.1750-2659.2009.00072.x pmid: 19496844

29. Hauge $\mathrm{SH}$ et al. Oseltamivir-resistant influenza viruses $A(H 1 N 1)$, Norway, 2007-08. Emerging Infectious Diseases, 2009, 15: 155-162. doi:10.3201/eid1502.081031 pmid:19193257

30. Sheu TG et al. Surveillance for neuraminidase inhibitor resistance among human influenza $A$ and $B$ viruses circulating worldwide from 2004 to 2008. Antimicrobial Agents and Chemotherapy, 2008, 52:3284-3292. doi:10.1128/AAC.00555-08 pmid: 18625765

31. Boltz DA et al. Emergence of H5N1 avian influenza viruses with reduced sensitivity to neuraminidase inhibitors and novel reassortants in Lao People's Democratic Republic. Journal of General Virology, 2010, 91:949-959. doi:10.1099/ vir.0.017459-0 pmid:20016036

32. Hurt AC et al. Increased detection in Australia and Singapore of a novel influenza $\mathrm{A}(\mathrm{H} 1 \mathrm{~N} 1) 2009$ variant with reduced oseltamivir and zanamivir sensitivity due to a S247N neuraminidase mutation. Euro Surveillance: European Communicable Disease Bulletin, 2011, 16(23):19909. pmid: 21679678

33. Submission to the Medicines Classification Committee for: oseltamivir (Tamiflu ${ }^{\circledR}$ powder filled capsules, 75mg). Review of the exemption to allow Pharmacy Prescribing of oseltamivir for treatment of influenza in adults and adolescents during the influenza season in New Zealand. Roche Products (New Zealand) Limited, $2008 \quad$ (http://www.medsafe.govt.nz/profs/class/ agen39Tamiflu.doc, accessed 28 November 2012).

34. Calatayud L et al. Oseltamivir-resistant pandemic (H1N1) 2009 virus infection in England and Scotland, 2009-2010. Emerging Infectious Diseases, 2011, 17:1807-1815. doi:10.3201/ eid1710.110117 pmid:22000349

35. Tashiro M et al.; Neuraminidase Inhibitor Susceptibility Network. Surveillance for neuraminidase-inhibitor-resistant influenza viruses in Japan, 1996-2007. Antiviral Therapy, 2009, 14:751-761. doi:10.3851/IMP1194 pmid:19812437

36. Eichner $\mathrm{M}$ et al. Antiviral prophylaxis during pandemic influenza may increase drug resistance. BMC Infectious Diseases, 2009, 9:4. doi:10.1186/1471-2334-9-4 pmid:19154598

37. Lipsitch $\mathrm{M}$ et al. Antiviral resistance and the control of pandemic influenza. PLoS Medicine, 2007, 4:e15. doi:10.1371/journal. pmed.0040015 pmid:17253900 